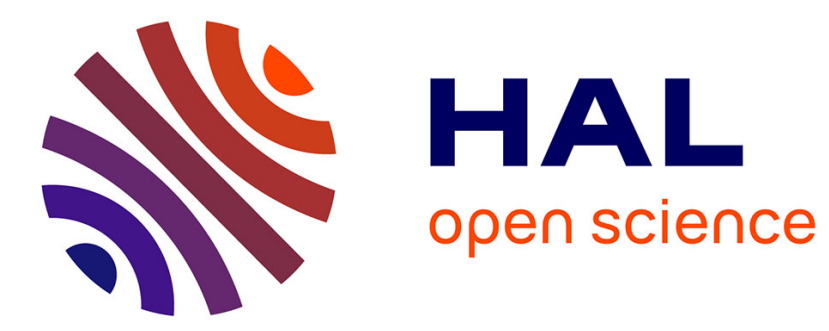

\title{
À la recherche des parts de marché perdues
}

Maria Bas, Lionel Fontagné, Philippe Martin, Thierry Mayer

\section{To cite this version:}

Maria Bas, Lionel Fontagné, Philippe Martin, Thierry Mayer. À la recherche des parts de marché perdues. [Rapport de recherche] 2015-23, Conseil d'Analyse Economique. 2015. hal-01299870

\section{HAL Id: hal-01299870 \\ https://hal.science/hal-01299870}

Submitted on 13 May 2016

HAL is a multi-disciplinary open access archive for the deposit and dissemination of scientific research documents, whether they are published or not. The documents may come from teaching and research institutions in France or abroad, or from public or private research centers.
L'archive ouverte pluridisciplinaire HAL, est destinée au dépôt et à la diffusion de documents scientifiques de niveau recherche, publiés ou non, émanant des établissements d'enseignement et de recherche français ou étrangers, des laboratoires publics ou privés. 


\section{À la recherche des parts de marché perdues}

\section{Les notes du conseil d'analyse économique, n²3, mai 2015}

\section{$\mathbf{L}$} 'arrivée sur les marchés mondiaux de nouvelles puissances - au premier rang desquelles la Chine fait mécaniquement diminuer les parts de marché des économies avancées. Mais la part de marché de la France à l'exportation diminue davantage que celles des autres pays européens. Ceci n'est pas le résultat d'une mauvaise spécialisation géographique ou sectorielle, d'une insuffisance des aides aux exportateurs, d'une sous-représentation des PME à l'export ou encore de contraintes de crédit, mais plus fondamentalement d'un " rapport qualité-prix » insuffisant des produits français, en moyenne. Lorsque la qualité est là, les résultats sont exceptionnels, comme le montrent le luxe, l'aéronautique ou les appareils de distribution électrique, autant de secteurs portés par un vaisseau amiral ou deux et/ou par des marques qui semblent jouer un rôle-clé.

La compétitivité d'un pays a une dimension prix et une dimension hors prix. S'agissant de la compétitivité prix, le coût direct du travail ne représente que $23 \%$ en moyenne de la valeur des exportations françaises et $44 \%$ en incluant le coût du travail des consommations intermédiaires domestiques. La compétitivité prix n'est donc pas uniquement l'affaire de coût du travail dans les entreprises exportatrices. Elle doit se chercher du côté des intrants, que ce soient des biens intermédiaires (éventuellement importés), de l'énergie ou bien des services produits en France pour les entreprises exportatrices. Le message central ici est que la compétitivité est l'affaire de tous, et non pas seulement celle des entreprises industrielles. Une meilleure efficacité des secteurs abrités de la concurrence internationale (services aux entreprises, construction, services publics) participe ainsi à la construction de la compétitivité prix.

La compétitivité hors prix est plus difficile à mesurer, nous nous appuyons sur des données désagrégées afin d'apporter un diagnostic par produit, approche microéconomique particulièrement adaptée pour révéler des effets qualité. Parmi les pays de l'OCDE, la France se maintient dans une relative bonne position si l'on compte le nombre des secteurs faisant partie des dix meilleurs en compétitivité hors prix ( $7^{\mathrm{e}}$ rang). Cependant, on observe un décrochage depuis 2008 avec le recul de plusieurs secteurs.

Politiques de compétitivité hors prix et politiques de productivité se confondent en grande partie, c'est pourquoi nous mettons en avant l'importance des réallocations de facteurs de production (travail et capital) pour faire plus rapidement émerger les entreprises les plus productives. Les réformes réduisant les réglementations sur les marchés des biens, des services et du travail devraient prendre explicitement en compte cet objectif. Par ailleurs, l'exemple du luxe montre l'importance des marques dans la construction d'une compétitivité hors prix. La protection de la propriété intellectuelle devrait constituer une priorité de la France et de I'Union européenne dans les négociations internationales. 


\section{Comment la France perd des parts de marché}

L'irruption des économies dites « émergentes » dans le commerce mondial a fait mécaniquement baisser les parts de marché de toutes les économies avancées. La baisse tendancielle des parts de marché de la France, documentée par de multiples rapports, est donc pour partie le reflet de la recomposition du commerce mondial. On peut néanmoins s'alarmer du fait que les parts de marché françaises reculent davantage que celles des autres pays de l'Union européenne, à l'exception de l'Italie (graphique 1) ${ }^{1}$. Entre 1995 et 2013, la part de marché de la France a reculé de $42 \%$ pour les biens et services, comme en Italie, alors que la baisse n'a été que de $21 \%$ pour l'Allemagne et $27 \%$ pour le Royaume-Uni, l'Espagne conservant une part de marché quasi stable ${ }^{2}$.

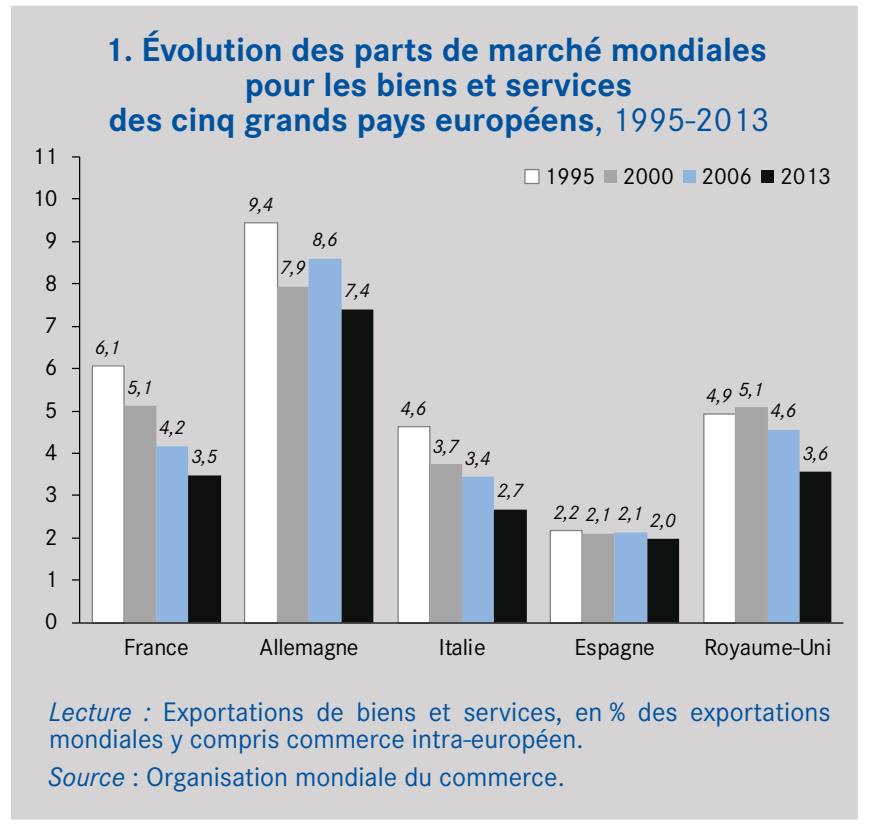

Nous nous intéressons dans cette Note aux seules exportations de biens qui forment l'essentiel du commerce international et pour lesquelles des statistiques détaillées sont disponibles par produit et par partenaire ${ }^{3}$.

\section{Le constat}

On attribue parfois la mauvaise performance française à l'exportation de biens à un mauvais positionnement géographique ou sectoriel. Selon cette logique, il faudrait, pour ralentir l'érosion des parts de marché, mieux identifier les pays et secteurs porteurs : grandes économies émergentes, agroalimentaire, santé, nouvelles technologies de l'information et de la communication, ville durable ${ }^{4}$.

Les pays européens, qui échangent beaucoup entre eux, souffrent d'un effet de composition géographique négatif, puisque l'Europe croît moins vite que le reste du monde (l'Asie en particulier). Aucun pays européen n'échappe à cet effet et la France se situe de ce point de vue dans la moyenne sur la période 2006-2014 (tableau 1 et méthode en encadré 1). Les pays qui ont réussi à mieux se positionner sur les marchés émergents souffrent légèrement moins de cet effet « européen » : c'est le cas de l'Allemagne et de l'Italie. Cependant l'effet géographique pénalise davantage l'Espagne et le Royaume-Uni que la France.

Quant à la spécialisation par produits, elle compte en moyenne relativement peu dans les variations de parts de marché observée entre 2006 et 2014, comme l'indique le tableau 1. Cependant, dans le cas français, cette spécialisation s'est avérée être un atout comparativement à l'Allemagne : le désavantage géographique des exportateurs français par rapport aux allemands a été surcompensé par un positionnement en produits plus favorable. La contribution combinée de la spécialisation pays et de la spécialisation produits pour expliquer les pertes de parts de marché est quasi nulle dans le cas de la

\begin{tabular}{|c|c|c|c|c|c|}
\hline \multicolumn{6}{|c|}{$\begin{array}{l}\text { 1. Évolution des parts de marché mondiales } \\
\text { pour les biens, 2006-T1 à 2014-T3 } \\
\text { variation annuelle moyenne en } \%\end{array}$} \\
\hline & \multirow{2}{*}{ 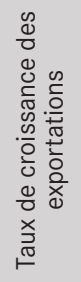 } & \multirow[b]{2}{*}{ 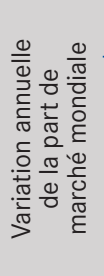 } & \multicolumn{3}{|c|}{ dont } \\
\hline & & & 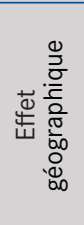 & 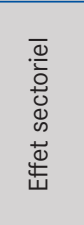 & 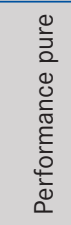 \\
\hline UE-28 & 4,4 & $-1,9$ & $-0,8$ & $-0,1$ & $-1,0$ \\
\hline Zone euro à 17 & 4,3 & $-2,0$ & $-0,8$ & $-0,1$ & $-1,1$ \\
\hline France & 3,0 & $-3,2$ & $-0,8$ & 0,7 & $-3,1$ \\
\hline Allemagne & 4,5 & $-1,7$ & $-0,5$ & $-0,2$ & $-1,0$ \\
\hline Italie & 4,0 & $-2,2$ & $-0,5$ & $-0,6$ & $-1,1$ \\
\hline Espagne & 4,5 & $-1,7$ & $-1,1$ & $-0,1$ & $-0,5$ \\
\hline Royaume-Uni & 2,3 & $-3,9$ & $-0,9$ & 0,4 & $-3,3$ \\
\hline Monde & 6,2 & 0,0 & 0,0 & 0,0 & 0,0 \\
\hline
\end{tabular}

Lecture: Les variations sont en delta-log. Par exemple, lorsque la part de marché passe de 10 à $9 \%$, la variation enregistrée est $\ln (9 / 10)=-10,5 \%$.

Source : Banque mondiale, Export Competitiveness Database.

Cette Note a bénéficié de plusieurs entretiens auprès d'organismes. Sans engager leur responsabilité, les auteurs remercient leurs interlocuteurs pour leur disponibilité et leurs contributions, plus particulièrement ceux de Business France, BPI France, la Direction générale du Trésor. Ils remercient également Alice Keogh, assistante de recherche au CAE, pour son aide précieuse.

${ }^{1}$ Dans cette Note, nous raisonnons sur les parts de marché mondiales, y compris les flux intra-européens. La part de marché de la France est le rapport entre les exportations françaises vers tous les marchés étrangers (y compris européens) et l'ensemble des exportations mondiales (y compris intra-européennes).

${ }^{2}$ La baisse des parts de marché française atteint $47 \%$ pour les seuls biens (entre 1995 et 2014 ), contre $21 \%$ pour l'Allemagne, 38 \% pour l'Italie et $41 \%$ pour le Royaume-Uni. Notons toutefois que la performance française se situe dans la moyenne de l'OCDE.

${ }^{3}$ Pour une analyse des exportations de services, voir par exemple Gaulier G., E. Milet et Mirza D. (2010) : «Les firmes françaises dans le commerce international des services ", Économie et Statistique, $\mathrm{n}^{\circ}$ 435-436, pp. 125-147.

${ }^{4}$ Voir Direction générale du Trésor (2012): Analyse prospective des marchés à l'export, par secteur et par pays, ministère de l'Économie et des Finances, octobre. 
France $(-0,1 \%$ par an en moyenne) tandis qu'elle est nettement négative pour l'Allemagne (- 0,7 \% par an). La meilleure performance de l'Allemagne s'explique donc exclusivement par des effets de compétitivité «pure » : les exportateurs français sont moins performants que les allemands pour vendre le même produit sur le même marché.

Cet effet de compétitivité "pure » est quantitativement important. Pour le voir, on peut calculer quelle aurait été l'évolution de la part de marché française si la France n'avait pas perdu davantage de compétitivité "pure » que l'Allemagne sur la période 2006-2014. La perte de part de marché aurait alors été de 0,75\% par an en moyenne, au lieu des $3 \%$ de pertes figurant dans le tableau 1. Cela représente un manque à gagner, en termes d'exportation de biens, de 112 milliards d'euros pour l'année $2014^{5}$.

Le commerce mondial a connu un recul très marqué en 2009 , suivi d'un ralentissement plus durable après le rebond mécanique de 2010. L'année 2009 marque aussi le début de la crise économique strictement européenne, qui a défavorisé encore un peu plus les exportateurs de l'Union européenne, fortement tournés sur les marchés de I'Union. Le tableau 2 montre que, sur la période 2009-2014, la contribution de la spécialisation géographique aux pertes de part de marché a environ doublé pour tous les pays européens. En France, toutefois, cet effet négatif a été compensé par un effet fortement positif de la spécialisation produits. Ainsi les pertes de parts de marché de la France depuis 2009 (- 1\% par an en moyenne) s'expliquent-elles, comme depuis 2006, essentiellement par un effet de compétitivité. Ces évolutions globales recouvrent toutefois des réalités très différentes. Un secteur particulier - le luxe - a enregistré de bonnes performances commerciales et mérite une analyse attentive.

\begin{tabular}{|c|c|c|c|c|c|}
\hline \multicolumn{6}{|c|}{$\begin{array}{l}\text { 2. Évolution des parts de marché mondiales } \\
\text { pour les biens de } 2009-\mathrm{T} 1 \text { à } 2014-\mathrm{T} 3 \\
\text { variation annuelle en } \%\end{array}$} \\
\hline & \multirow{2}{*}{ 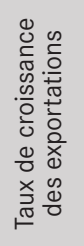 } & \multirow{2}{*}{ 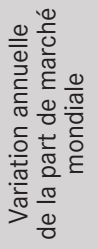 } & \multicolumn{3}{|c|}{ dont } \\
\hline & & & 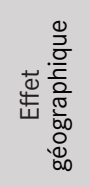 & 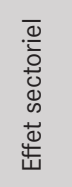 & 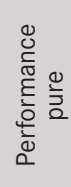 \\
\hline UE-28 & 0,3 & $-2,3$ & $-1,8$ & 0,5 & $-1,0$ \\
\hline Zone euro à 17 & 0,1 & $-2,5$ & $-1,8$ & 0,5 & $-1,3$ \\
\hline France & $-1,0$ & $-3,5$ & $-1,5$ & 1,5 & $-3,5$ \\
\hline Allemagne & 0,5 & $-2,0$ & $-1,3$ & 0,5 & $-1,2$ \\
\hline Italie & $-0,3$ & $-2,8$ & $-1,4$ & 0,2 & $-1,6$ \\
\hline Espagne & 1,1 & $-1,4$ & $-1,9$ & 0,7 & $-0,1$ \\
\hline Royaume-Uni & $-0,2$ & $-2,7$ & $-1,6$ & 0,7 & $-1,8$ \\
\hline Monde & 2,5 & 0,0 & 0,0 & 0,0 & 0,0 \\
\hline
\end{tabular}

\begin{abstract}
Constat 1. Les mauvaises performances françaises à l'exportation sont liées à un « rapport qualité-prix » insuffisant, non à un mauvais positionnement pays ou produits.
\end{abstract}

\section{Décomposition des variations de part de marché entre 2006 et 2014}

Afin d'isoler la contribution des spécialisations géographique et sectorielle à la variation des parts de marché, nous utilisons les données de la base Export Competitiveness Database développée par la Banque mondiale, en collaboration avec la Banque de France et le Centre du commerce international (CNUCEDOMC). La méthode dite "shift share " proposée par Gaulier et al. (2013) a y est appliquée à des données trimestrielles, pour une période commençant en 2006, pour 228 pays ou territoires, au niveau HS6 de la nomenclature harmonisée des échanges, soit environ 5000 produits. Les dernières données disponibles correspondent au $3^{\mathrm{e}}$ trimestre 2014. Par rapport à la méthode "shift-share traditionnelle $\|^{b}$, celle utilisée pour la construction de cette base bénéficie d'une plus grande fréquence des données et d'une prise en compte de la marge extensive du commerce (augmentation du nombre de produits exportés ou du nombre de destinations servies).

La variation des exportations pour chaque catégorie de produit/pays exportateur/pays importateur/trimestre, est régressée sur des effets fixes produit, exportateur et importateur, permettant par différence d'isoler l'effet de compétitivité " pure " pour chaque pays exportateur. Cet effet de compétitivité "pure " répond à la question suivante: "quelle aurait été la variation des exportations d'un pays si la structure géographique et sectorielle de ses exportations avait été identique à celle de ses concurrents? $\|^{c}$.

a Gaulier G., G. Santoni, D. Taglioni et S. Zignago (2013): « In the Wake of the Global Crisis: Evidence from a New Quarterly Database of Export Competitiveness ", World Bank Policy Research Working Paper, $n^{\circ} 6733$.

b Cheptea A., G. Gaulier et S. Zignago (2005) : « World Trade Competitiveness: A Disaggregated View by Shift-Share Analysis ", Document de Travail du CEPII, $\mathrm{n}^{\circ}$ 2005-23. Cheptea A., C. Emlinger, L. Fontagné, G. Orefice et O. Pindyuk (2014) : «The Development of EU and EU Member States' External Competitiveness ", Document de Travail du CEPII, n 2014-06. Cheptea A., L. Fontagné et S. Zignago (2014), "European Export Performance ", Review of World Economics, vol. 150, $\mathrm{n}^{\circ}$ 1, pp. 25-58.

${ }^{c}$ Nous remercions Gianluca Santoni (CEPII) pour l'extraction des données.

\footnotetext{
${ }^{5}$ Ce calcul est fondé sur la variation comparée des parts de marché de l'Allemagne et le la France jusqu'au 4e trimestre 2013. On applique le " déficit ॥ d'exportations ainsi mesuré à la fin de 2013 aux échanges mondiaux en 2014 ; soit 0,76 point de 18784 x 1,021/1,3 avec des exportations mondiales de marchandises de 18784 milliards de dollars en 2013 et une croissance estimée de 2,1\% du commerce mondial en valeur en 2014 (estimation CPB, World Trade Monitor). Nous prenons un taux de change moyen de 1,3 entre l'euro et le dollar.
} 


\section{Le contre-exemple du luxe}

Industrie à fort contenu en main d'œuvre localisée - au moins pour partie - dans un pays réputé souffrir de coûts salariaux élevés, le luxe français est parvenu à concurrencer les pays émergents et à tirer parti de la rapide croissance en leur sein d'une classe aisée. Deux études récentes permettent de comprendre ce succès à partir de données issues d'un regroupement d'une partie des industries du luxe au sein du Comité Colbert ${ }^{6}$. Les secteurs couverts sont assez variés : arts de la table, décoration, vêtements, boissons, parfums, bijoux, sacs et chaussures, confiserie.

Trois résultats apparaissent :

- les pays avancés sont moins désavantagés, toutes choses égales par ailleurs, pour l'exportation des produits de luxe que pour les autres produits issus de l'industrie : le positionnement haut de gamme les «protège » de la concurrence des pays émergents ;

- les exportations de produits de luxe sont moins sensibles à la distance que la moyenne des autres produits ;

- les exportations de produits de luxe réagissent davantage que les autres exportations à la hausse du pouvoir d'achat dans les marchés de destination, mais ceci ne vaut que pour une poignée de pays exportateurs, dont la France.

$\mathrm{Au}$ cœur de ce succès, une variable déterminante: les marques. Le facteur explicatif distinguant la France (mais aussi l'Italie et la Suisse) est le nombre de marques de luxe du pays exportateur parmi les 100 premières marques mondiales : 24 en France, contre 10 en Suisse, 7 en Italie et seulement 1 au Japon (cf. World Luxury Association).

Constat 2. Les secteurs ayant le mieux résisté s'appuient sur des marques fortes.

\section{Trop peu d'exportateurs ?}

On a vu plus haut comment l'on peut décomposer la croissance des exportations en une composante de spécialisation géographique, une composante de spécialisation sectorielle et un effet de compétitivité " pure ॥. Une autre décomposition intéressante distingue l'amplification, au cours du temps, des flux déjà existants (par exemple, les exportations depuis la France de petites voitures Peugeot vers le Danemark), de la création de nouveaux flux (nouveaux produits, nouveaux marchés ou nouvelles entreprises exportatrices).

À court terme (d'une année sur l'autre), la croissance des exportations françaises s'explique de manière écrasante par l'amplification de flux déjà en place, ce que l'on appelle la marge intensive $(87,7 \%)^{7}$. Le reste $(12,3 \%)$, appelé marge extensive, correspond à la création de nouveaux flux : $2,4 \%$ sont le fait de l'arrivée de nouvelles entreprises (nette du départ d'entreprises antérieurement présentes à l'exportation) ; et 9,9\%, viennent d'entreprises déjà présentes mais qui ajoutent ou retirent des produits et/ou des marchés de destination à leur portefeuille d'exportation. À court terme, ce n'est donc pas l'arrivée de nouvelles entreprises exportatrices qui explique principalement la croissance des exportations françaises.

Sur une période de dix ans, en revanche, la marge extensive explique $53,5 \%$ de la dynamique des exportations : $26,2 \%$ du fait de l'entrée de nouvelles entreprises et $27,3 \%$ du fait de la multiplication des produits ou marchés par celles déjà en place. À cet horizon, la marge intensive n'explique que $46,5 \%$ de la croissance des exportations agrégées. La différence entre l'analyse à court terme et à l'horizon d'une décennie tient au fait que les nouveaux flux d'exportation sont de petite taille, mais sont susceptibles de croître rapidement au cours du temps s'ils perdurent (en raison d'une sélection drastique des meilleurs entrants, les autres sortant assez vite du marché), si bien qu'au bout de dix ans, ces flux initialement faibles deviennent significatifs.

La piètre performance française à l'exportation ne résulte pas d'un partage différent entre le développement des marchés existants (qui se serait maintenu) et la conquête de nouveaux marchés (qui aurait été insuffisante - voir la chute du nombre d'entreprises exportatrices entre 2000 et 2009, graphique 2). La très forte concentration des exportations françaises sur un petit nombre d'exportateurs est un phénomène caractéristique des économies avancées ${ }^{8}$, et le partage de la dynamique des exportations entre marges intensive et extensive est similaire dans d'autres pays. Par ailleurs, la performance française n'est pas due à une sous-représentation particulière des PME dans nos exportations : un tiers de la valeur des exportations françaises hors Union européenne est le fait de PME en 2011. C'est moins que l'Italie (49\%) mais plus que l'Allemagne $(28 \%)$ et dans la moyenne européenne ${ }^{9}$.

\footnotetext{
${ }^{6}$ Cf. www.comitecolbert.com. Voir Martin J. et F. Mayneris (2015) : «High-End Variety Exporters Defying Distance: Micro Facts and Macroeconomic Implications ", Journal of International Economics, à paraître et Fontagné L. et S. Hatte (2014) : "European High-End Varieties in International Competition », Document de Travail du CEPII, $\mathrm{n}^{\circ}$ 2014-27. Les résultats de ces deux études sont confirmés dans le cas particulier du vin de Champagne par Crozet M. K. Head et T. Mayer (2012) : "Quality Sorting and Trade: Firm-Level Evidence for French Wine », Review of Economic Studies, vol. 79 , ${ }^{\circ}{ }^{2}$, pp. $606-644$.

${ }^{7}$ Voir Berman N., V. Rebeyrol et V. Vicard (2015) : “Demand Learning and Exporter Dynamics », Document de Travail de la Banque de France, $n^{\circ} 551$, mai.

${ }^{8}$ En 2003, les $5 \%$ plus gros exportateurs français réalisaient $73 \%$ des exportations agrégées du pays. Le chiffre correspondant était de $81 \%$ pour l'Allemagne et 69 \% pour le Royaume-Uni, I'Italie se situant un peu en dessous à 59 \%. Voir Mayer T. et G. Ottaviano (2007) : « The Happy Few: The Internationalisation of European Firms. New Facts Based on Firm-Level Evidence ", Bruegel Blueprint Series, vol. III. Cette concentration extrême reste vraie à un niveau encore plus fin : en 2008, les dix premiers exportateurs français représentaient un peu plus de $20 \%$ des exportations totales ; en Espagne ce chiffre dépassait les 40 \% et en Italie environ 10 \%, voir Berthou A., E. Dhyne, M. Bugamelli, A-M. Cazacu, C.V. Demian, C-V., P. Harasztosi, T. Lalinsky, J. Meriküll, F. Oropallo et A.C. Soares (2015) : « Assessing European Firms' Exports and Productivity Distributions: The CompNet Trade Module », ECB Working Paper, ${ }^{\circ}$ 1788, mai.

${ }^{9}$ Voir Cernat L, A. Norman-López et A. Dutch T-Figueras (2014) : « SMEs Are More Important than You Think! Challenges and Opportunities for EU Exporting SMEs », Chief Economist DG TRADE Note, $\mathrm{n}^{\circ} 3$, septembre.
} 


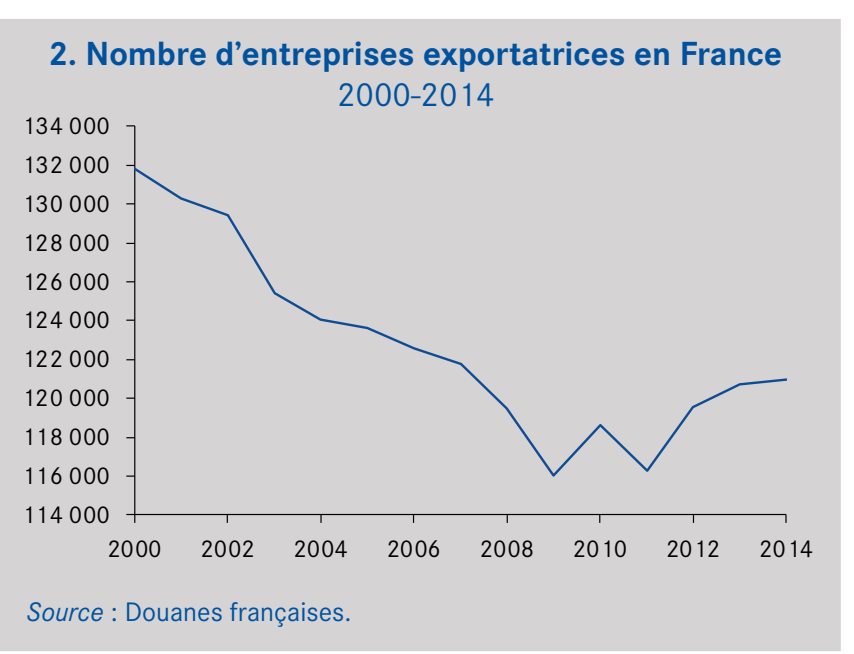

\section{Les politiques de soutien à l'exportation}

Comme on l'a vu, la création de nouveaux flux d'exportation est un élément déterminant de la dynamique des exportations, si ce n'est à court terme, du moins à l'horizon de dix ans. Ces nouveaux flux peuvent venir de grandes entreprises développant de nouveaux produits ou investissant de nouveaux marchés. Ils peuvent aussi venir de PME primoexportatrices. Trois raisons peuvent justifier l'intervention publique dans ce domaine.

\section{Les coûts liés à la recherche d'information}

Selon les théories récentes du commerce international ${ }^{10}$, l'entreprise primo-exportatrice fait face à un coût fixe de recherche d'information, qui pèse proportionnellement plus sur une petite entreprise que sur une grande. De nombreuses PME n'exportent pas car leur productivité est insuffisante pour couvrir ce coût fixe. La puissance publique peut alors souhaiter soutenir les PME les plus proches du seuil de productivité pour leur permettre d'exporter, sachant que le coût d'entrée n'est supporté qu'une seule fois. L'action publique est cependant confrontée à plusieurs difficultés (encadré 2) : il est difficile de repérer les entreprises proches du seuil de productivité leur permettant d'exporter; un grand nombre d'entreprises primo-exportatrices ne parviennent pas à se maintenir sur les marchés cibles, ce qui réduit d'autant le rendement de l'intervention publique ${ }^{11}$; si la multiplicité des dispositifs de soutien à l'exportation conduit à remplacer un coût d'entrée sur un marché étranger (subventionné par la puissance publique) par un coût de recherche du dispositif de soutien adéquat (supporté par la PME), l'effet net sur la capacité à exporter pourrait être incertain. De fait, la complexité des dispositifs en France est régulièrement pointée par les missions d'évaluation, malgré les efforts de rationalisation ${ }^{12}$.

Quoi qu'il en soit, la politique d'aide aux exportations telle qu'elle est structurée aujourd'hui ne peut suffire à expliquer la dégradation du solde commercial français. Les entreprises concernées sont généralement de petite taille et contribuent modestement au commerce extérieur; en outre, le commerce extérieur s'est dégradé alors même que les dispositifs d'appui étaient améliorés. L'effort significatif consenti par la puissance publique (estimé à environ 600 millions d'euros en 2012) ${ }^{13}$ a été récemment réorienté des primoexportateurs vers les entreprises ayant déjà une activité internationale, en visant l'augmentation de leurs exportations et du nombre de leurs filiales à l'étranger.

\section{Les imperfections du marché du crédit}

Une deuxième justification de l'intervention publique a trait aux contraintes de financement que subissent les PME, plus particulièrement lorsqu'elles investissent pour atteindre des marchés d'exportation. L'évaluation de ces interventions en termes de financement pose les mêmes difficultés que celle des interventions en termes d'accompagnement (identification du groupe de référence).

Si ces interventions publiques en termes de financement touchent une proportion non négligeable des entreprises exportatrices (voir encadré 2), il est peu probable que le financement soit l'élément décisif pour redresser les parts de marché françaises. Selon Bricongne et al. (2012) ${ }^{14}$, durant la crise financière mondiale de 2008, les entreprises exportatrices françaises ont été touchées moins par l'arrêt du crédit que par la chute des échanges internationaux : plus de $60 \%$ de la baisse des exportations françaises est à mettre au compte de la « marge intensive nette » des très grandes entreprises exportatrices (« top $1 \%$ »), qui ont été peu touchées par les contraintes de crédit. L'effet direct du resserrement du crédit a été ressenti par les entreprises déjà en difficulté financière, pour lesquelles les contraintes de prêts liées à la crise ont été un facteur aggravant ; cependant, leur nombre étant réduit, l'impact reste limité.

\section{Les externalités}

Une dernière justification des aides à l'exportation repose sur la présence d'effets externes positifs sur les entreprises environnantes, en amont ou en aval de l'entreprise exportatrice. Permettre à une entreprise d'exporter a des effets multiplicateurs dans la mesure où elle pourra partager un certain

\footnotetext{
${ }^{10}$ Mélitz M. (2003) : « The Impact of Trade on Intra-Industry Reallocations and Aggregate Industry Productivity », Econometrica , vol. 71 , $\mathrm{n}^{\circ}$ 6, pp. $1695-1725$.

${ }^{11}$ Selon Bentejac et Desponts (2013), environ un tiers des primo-exportateurs (qui n'ont pas exporté dans les cinq dernières années) survit au-delà d'un an. La proportion est de $70 \%$ pour les entreprises adossées à un groupe. Voir Bentejac A. et J. Desponts (2013) : Mission d'évaluation sur l'efficacité du dispositif d'appui à l'internationalisation de l'économie française, ministère du Commerce extérieur, La Documentation française, juin.

${ }^{12}$ Voir Bentejac et Desponts (2015), op. cit., Cour des Comptes (2011) : Le soutien public aux entreprises exportatrices, Rapport annuel et Inspection générale des Finances (2013) : « Pour des aides simples et efficaces au service de la compétitivité ॥, Rapport de l'IGF, n²013-M-016-02, juin.

${ }^{13}$ Voir Bentejac et Desponts (2013) op. cit.

${ }^{14}$ Bricongne J.-Ch., L. Fontagné, G. Gaulier, D. Taglioni et V. Vicard (2012) : "Firms and the Global Crisis: French Exports in the Turmoil », Journal of International Economics, vol. 87, $\mathrm{n}^{\circ} 1$, pp. 134-146.
} 


\section{Les politiques publiques de soutien à l'exportation}

En France, l'appui à l'exportation mobilise un grand nombre d'intervenants et plus de $90 \%$ des ETI et PME déjà internationalisées ou en passe de l'être ( $2 \%$ du total des entreprises) déclarent avoir eu recours à au moins un organisme de soutien ${ }^{a}$. Les dispositifs couvrent deux grands types d'interventions :

- l'accompagnement: information, mise en relation avec des partenaires sur les marchés cibles, présence dans les salons professionnels, embauche de volontaires internationaux en entreprise... Environ 9000 entreprises sont accompagnées chaque année par Business France (anciennement Ubifrance), une action complétée par le réseau des chambres de commerce françaises à l'étranger, celui de la DG Trésor, le réseau diplomatique, celui des conseillers du commerce extérieur, auxquels s'ajoutent des agences sectorielles (agriculture, produits culturels), l'intervention des régions, celle des métropoles et les dispositifs supranationaux de promotion des exportations sous l'initiative de l'Union européenne ;

- le financement et l'assurance: l'intervention publique est aujourd'hui mise en œuvre essentiellement par BPI France et la Coface. BPI France propose trois dispositifs pour environ 1000 entreprises par an: prêts de développement export (prêts non gagés garantis par un fonds public) ; crédits de trésorerie (avances à l'exportation, crédit acheteur) ; garanties de prêts bancaires. La Coface quant à elle propose de l'assurance-crédit (en tant qu'assureur privé) ainsi qu'une assurance prospection (pour le compte de l'État). Son rapport d'activité de 2013 fait état de 37000 clients.
Crozet et al. (2013) ont évalué l'impact direct d'un nombre restreint de dispositifs proposés par Ubifrance et Coface sur les performances à l'exportation des entreprises françaises entre 2005 et 2010, en comparant les entreprises aidées aux entreprises non aidées ayant les mêmes caractéristiques en termes de secteur, taille et productivité. Selon l'étude, l'assurance prospection de la Coface semble principalement augmenter les volumes exportés des exportateurs déjà en place (+ $20 \%$ environ) et le nombre de destinations (environ un pays supplémentaire). Les mesures d'accompagnement collectif mises en place par Ubifrance semblent être les seules à promouvoir la décision d'exporter (elles ont un effet comparable à celles de la Coface sur les volumes exportés), mais cet effet semble de très courte durée, contrairement à l'assurance prospection qui a des effets plus durables. Ces résultats sont cohérents avec ceux que l'on obtient dans d'autres pays ${ }^{c}$, même s'il convient de noter une assez forte sensibilité des résultats au choix du groupe de contrôle dans toutes ces études, y compris celle sur la France. Les résultats sont aussi cohérents avec les enquêtes réalisées aussi bien par Business France que par BPI France.

${ }^{a}$ Enquête auprès de 250 PMEetETI, voirBentejac et Desponts (2013)op. cit. ${ }^{b}$ Crozet M., T. Mayer, F. Mayneris et D. Mirza (2013) : « Efficacité des dispositifs de soutien à l'exportation des firmes françaises $"$, Document de Travail du CEPII, n² 2013-03.

${ }^{c}$ Cadot O., A.M. Fernandes, J. Gourdon, J. et A. Mattoo (2011) : "An Evaluation of Tunisia's Export Promotion Program ॥, IGC-DECTI Workshop, The World Bank, 14 octobre. Volpe Martincus, C. et J. Carballo (2008) : « Is Export Promotion Effective in Developing Countries? Firm-Level Evidence on the Intensive and the Extensive Margins of Exports 1 , Journal of International Economics, vol. 76, $n^{\circ}$ 1, pp. 89-106. Van Biesebroeck J., E. Yu et S. Chen (2015) : "The Impact of Trade Promotion Services on Canadian Exporter Performance ॥, Canadian Journal of Economics, à paraitre. nombre d'informations avec d'autres exportateurs potentiels, ou bien simplement servir d'effet de démonstration pour des concurrents proches. Les études récentes confirment l'existence de tels effets positifs ${ }^{15}$. Cependant, les pôles de compétitivité ne semblent pas avoir d'effet d'entraînement sur la R\&D, le nombre de brevets ou le chiffre d'affaires. Certes, certains travaux mettent en évidence un effet positif des pôles de compétitivité sur la probabilité de continuer à exporter pour les exportateurs appartenant aux pôles. Ceux-ci sont cependant plus dépendants de l'entreprise " leader » du pôle et leur appartenance aux pôles ne semble pas les avoir protégées pendant la crise financière de 2008-2009 ${ }^{16}$.

Par rapport aux autres pays européens, la France se situe dans la fourchette haute des soutiens publics à l'exportation (encadré 3). La nature des dispositifs de soutien est similaire d'un pays à l'autre. Toutefois, les dispositifs français se caractérisent par une part plus importante des services facturés à l'entreprise.

\begin{abstract}
Constat 3. Les PME françaises disposent d'une palette large de soutiens publics à l'exportation. Même s'ils pourraient encore utilement être rationalisés, ces dispositifs ne sont pas la solution principale à l'érosion des parts de marché françaises.
\end{abstract}

\section{Une compétitivité hors prix insuffisante}

La compétitivité " pure » définie plus haut a une dimension prix et une dimension hors prix. La compétitivité prix est la résultante des coûts unitaires de production (coût du travail, énergie, coût du capital, consommations intermédiaires... nets de gains de productivité), des marges, du taux de change nominal. La compétitivité hors prix peut être considérée comme un résidu au sens où elle est la partie de la demande non expliquée par le prix. Elle comporte une dimension variété (différenciation horizontale des produits, par exemple,

\footnotetext{
${ }^{15}$ Koenig P., F. Mayneris et S. Poncet (2010) : « Local Export Spillovers in France », European Economic Review, vol. 54, n 4, pp. $622-641$.

${ }^{16}$ Voir Askenazy P. et P. Martin (2014) : « Promouvoir l'égalité des chances à travers le territoire », Note du Conseil d'analyse économique, $\mathrm{n}^{\circ} 20$, février, qui recommandent une évaluation indépendante des pôles de compétitivité et une réduction de leur nombre, et Bellégo C. et V. Dortet-Bernadet (2013) : « La participation aux pôles de compétitivité : quelle incidence sur les dépenses de R\&D et l'activité des PME et ETI ? ॥, Document de Travail de l'INSEE, $\mathrm{n}^{\circ}$ G2013/06.
} 


\section{Les politiques de soutien à l'exportation dans quelques pays européens}

En Allemagne, la promotion du commerce extérieur est pilotée pour l'essentiel par le ministère de l'Économie, avec un budget de 138,4 millions d'euros en 2012. L'essentiel est investi dans l'organisation de foires et salons (40\%) et dans le réseau consulaire des 120 chambres de commerce bilatérales $(27 \%)$. Ces dernières totalisent 2200 agents et 50000 entreprises adhérentes, répartis sur 80 pays. Le dispositif de soutien au niveau fédéral s'appuie également sur les conseillers économiques des ambassades et sur le GTAI (Germany Trade and Invest), agence du ministère de l'Économie comptabilisant environ 300 agents et un budget de 17 millions d'euros. Les entreprises sont par ailleurs appuyées au niveau des Länder à travers les chambres de commerce locales ; les Länder les plus industrialisés ont également des organismes propres d'accompagnement de leurs entreprises vers l'étranger. Par ailleurs, les entreprises bénéficient de garanties et d'assurance-crédit (30 milliards d'euros 2011), dont $75 \%$ sont affectés au commerce avec les pays émergents et en voie de développement; ce budget sert à soutenir les exportations, mais peut aussi recouvrir des objectifs de politique industrielle, de politique étrangère ou de sauvegarde de l'emploi.

Le système italien s'appuie sur le ministère des Affaires étrangères et sur le ministère du Développement économique ; le premier a un rôle de coordination grâce à son réseau diplomatique, tandis que le second se charge d'octroyer les aides à l'export (soutien aux actions promotionnelles, prêts bonifiés ou produits d'assurance-crédit, principalement en faveur des PME). Ce système a été réformé en 2013 pour recentraliser certaines compétences qui avaient été dévolues aux régions, dans l'optique de rendre les dispositifs plus lisibles et plus efficaces. L'effort financier consenti par l'État italien a été réduit de moitié ces dernières années, atteignant 33 millions d'euros en 2012.

$\mathrm{Au}$ Royaume-Uni, le soutien aux exportations s'organise sous la cotutelle des ministères du Commerce et des Affaires étrangères, à travers l'agence UKTI (UK Trade and Investment - équivalent de Business France). Celle-ci repose sur un dispositif régional de douze agences dispersées à travers le pays. UKTI dispose d'un budget équivalent à 368 millions d'euros pour 2014-2015, soit plus du double du budget 2013-2014. Elle emploie près de 2300 agents, dont la moitié est localisée à l'étranger. Par ailleurs, le modèle britannique repose sur une collaboration avec des prestataires privés et les chambres de commerce à l'étranger (par exemple, le China Britain Business Council). Des priorités sectorielles (santé, défense, industrie culturelle, hydrocarbures et agroalimentaire) et géographiques (grand pays émergents) ont été définies. 54190 entreprises ont bénéficié d'un ou plusieurs services d'UKTI entre octobre 2013 et septembre 2014 (contre 25400 en 2011), dont 11430 primo-exportateurs. Ces derniers ont accès à de nombreuses prestations gratuites ou subventionnées, comme par exemple les conseils à l'export d'un expert UKTI, une aide financière pour assister à des salons et foires à l'étranger ou accéder à des analyses sur les marchés étrangers. D'autres prestations sont payantes, comme l'Overseas Market Introduction Service (ensemble évolutif de mesures recourant à des experts UKTI sur place) ou les Postgraduate Students for International Business, équivalent britannique des volontaires internationaux inauguré en 2014. À travers UK Export Finance, les entreprises n'ayant pas réussi à s'assurer sur le marché privé ont pu bénéficier en 2013 de produits assurantiels à hauteur de 2 milliards de livres sterling. des parfums différents de yaourts) et une dimension qualité (différenciation verticale des produits, par exemple, des yaourts plus ou moins onctueux).

Le fait que la compétitivité hors prix ne soit pas directement mesurable rend assez fragile le diagnostic que l'on peut poser, notamment lorsqu'on s'appuie sur des données agrégées ${ }^{17}$. Nous adoptons ici une perspective microéconomique : nous comparons l'évolution de la compétitivité hors prix des produits exportés par France à ceux des autres grands pays de l'OCDE, à partir de données désagrégées par produit sur la période 2000-2013. L'indicateur de compétitivité hors prix utilisé reflète les caractéristiques des produits qui augmentent leur demande à prix donné. L'idée est que les consommateurs sont prêts à payer un prix plus élevé pour des produits possédant d'autres attributs que le prix qu'ils valorisent fortement : marque, image de l'entreprise, du pays exportateur, qualité des services associés, réputation, fiabilité du produit, design, etc. La méthode est précisée dans l'encadré 4.

\section{La France au $7^{\mathrm{e}}$ rang de l'OCDE...}

On se limite ici aux secteurs représentant plus d'un pourcent des exportations de chaque pays considéré. Le tableau 3 indique les dix secteurs arrivant en tête pour leur compétitivité hors prix en France et en Allemagne en 2013. Les secteurs sont classés par rang décroissant au sein de l'OCDE. Ainsi, le premier secteur pour la France, en termes de compétitivité hors prix, est l'aéronautique, où la France est première parmi les pays de l'OCDE. Cette compétitivité hors prix

${ }^{17}$ Voir par exemple Borey G. et B. Quille (2013) : «Comment s'explique le rééquilibrage des balances commerciales en Europe ? », Note de Conjoncture de I'INSEE, juin, pp. 19-40. 


\section{Mesure de la compétitivité hors prix}

Bas, Martin et Mayer (2014) ont adapté la méthode développée par Khandelwal, Schott et Wei (2013) afin d'estimer la qualité des produits exportés au niveau HS6 (environ 5000 produits) en utilisant des données bilatérales de commerce international ${ }^{a}$. Les données utilisées ici sont celles de $\mathrm{BACl}$, une base développée par le CEPII à partir de la base COMTRADE des Nations unies ${ }^{b}$. Les estimations se focalisent sur les 50 pays exportateurs et importateurs qui ont les flux de commerce les plus élevés ${ }^{c}$. Afin de comparer des pays semblables à la France, nous nous concentrons sur les pays exportateurs appartenant à l'OCDE.

La compétitivité hors prix est estimée à partir d'une fonction de demande qui dépend négativement des prix, avec une élasticité de substitution constante, et positivement des attributs hors prix des produits (dont la qualité perçue) :

$$
x_{\text {kint }}+\sigma_{k} p_{\text {kint }}=\beta \mathrm{PIB}_{\text {it }}+\lambda D_{\text {in }}+\alpha_{\text {knt }}+\varepsilon_{\text {kint }}
$$

où $x_{\text {kint }}$ et $p_{\text {kint }}$ sont les logarithmes de la quantité et du prix (valeur unitaire) du produit $k$ exporté par le pays $i$ vers la destination $n$ l'année $t$.

La méthode a été amendée afin de prendre en compte les déterminants bilatéraux du commerce international comme la distance, la langue commune, les effets de frontière et les liens coloniaux, regroupés dans le terme $D_{\text {in }}$. Par ailleurs, l'effet fixe $\alpha_{\mathrm{knt}}$ prend en compte la demande ainsi que le degré de concurrence dans le pays de destination. Enfin, l'équation contrôle l'effet de la taille du pays d'origine. L'élasticité de substitution entre produits $\sigma_{k}$ est reprise de Broda et Weinstein (2006) ${ }^{d}$.

La compétitivité hors prix estimée $q_{\text {kint }}$ est le résidu de l'équation $\varepsilon_{\text {kint }}$ normalisé par l'élasticité du produit correspondant : $q_{\text {kint }}=\varepsilon_{\text {kint }} /\left(\sigma_{k}-1\right)$. Un même résidu révèle une compétitivité hors prix plus élevée s'il s'agit d'un produit à faible élasticité, pour lequel les écarts de prix jouent peu sur les ventes. Cette mesure de compétitivité hors prix est ensuite agrégée par secteurs (102 secteurs).

\footnotetext{
${ }^{a}$ Bas M., P. Martin et T. Mayer (2014) : Report on Main Directions of Research Towards Better Assessment of Competitiveness, Mapcompete. Khandelwal A., P. Schott et S. Wei (2013) : «Trade Liberalization and Embedded Institutional Reform: Evidence from Chinese Exporters ॥, American Economic Review, vol. 103, $\mathrm{n}^{\circ} 6$, pp. 2169-95.

${ }^{b}$ Pour une description détaillée de ces données voir Gaulier $\mathrm{G}$ et S. Zignago (2010) : « BACl: International Trade Database at the Product-Level, The 1994-2007 Version ॥, Document de Travail du CEPII, $\mathrm{n}^{\circ} 2010-23$

${ }^{c}$ La liste des pays est détaillée dans Bas, Martin et Mayer (2014) op. cit., tableau 1.

${ }^{d}$ Broda C. et D. Weinstein (2006) : «Globalization and the Gains from Variety ", Quarterly Journal of Economics vol. $121, n^{\circ} 2$, mai.
}

est évaluée à 2,4 : dans ce secteur, les exportateurs français peuvent avoir des prix plus de deux fois supérieurs à ce qu'ils seraient si leur qualité était identique à la moyenne des pays de l'OCDE. L'avantage atteint 7,3 dans le secteur de la maroquinerie, où la France se situe au deuxième rang des pays de l'OCDE en termes de compétitivité hors prix.

\section{Les dix premiers secteurs pour la compétitivité} hors prix France et Allemagne, 2013

\begin{tabular}{|c|c|c|c|c|}
\hline & $\begin{array}{c}\text { Part de } \\
\text { marché } \\
\text { dans } \\
\text { I'OCDE, } \\
\text { en \% }\end{array}$ & $\begin{array}{c}\text { Part du } \\
\text { secteur } \\
\text { dans les } \\
\text { expor- } \\
\text { tations } \\
\text { totales du } \\
\text { pays, en } \%\end{array}$ & $\begin{array}{c}\text { Compé- } \\
\text { titivité } \\
\text { hors prix }\end{array}$ & $\begin{array}{l}\text { Rang } \\
\text { OCDE }\end{array}$ \\
\hline \multicolumn{5}{|l|}{ France } \\
\hline Aéronautique & 10,2 & 3,4 & 2,4 & 1 \\
\hline Maroquinerie & 25,6 & 1,3 & 7,3 & 2 \\
\hline Vin & 28,0 & 2,4 & 2,2 & 3 \\
\hline $\begin{array}{l}\text { Appareils de } \\
\text { distribution électrique }\end{array}$ & 6,0 & 1,7 & 4,5 & 3 \\
\hline $\begin{array}{l}\text { Pièces détachées } \\
\text { automobile }\end{array}$ & 6,2 & 6,0 & 1,4 & 5 \\
\hline Produits laitiers & 14,6 & 2,2 & 1,2 & 5 \\
\hline Vêtements & 9,3 & 1,1 & 1,2 & 5 \\
\hline Plastiques & 7,5 & 3,9 & 1,1 & 7 \\
\hline $\begin{array}{l}\text { Autres produits } \\
\text { métalliques, }\end{array}$ & 5,8 & 2,2 & 1,2 & 7 \\
\hline Produits plastiques & 6,4 & 2,8 & 1,3 & 8 \\
\hline \multicolumn{5}{|l|}{ Allemagne } \\
\hline $\begin{array}{l}\text { Pièces détachées } \\
\text { automobile }\end{array}$ & 22,6 & 8,0 & 3,4 & 1 \\
\hline Métaux non ferreux & 16,4 & 3,6 & 1,4 & 1 \\
\hline Produits plastiques & 20,4 & 3,3 & 2,8 & 1 \\
\hline Véhicules automobiles & 16,8 & 3,0 & 1,6 & 1 \\
\hline $\begin{array}{l}\text { Autres produits } \\
\text { métalliques }\end{array}$ & 21,5 & 3,0 & 2,2 & 1 \\
\hline $\begin{array}{l}\text { Appareils de } \\
\text { distribution électrique }\end{array}$ & 24,2 & 2,5 & 34,2 & 1 \\
\hline Machineries, autres & 20,7 & 2,3 & 3,7 & 1 \\
\hline Machines-outils & 27,4 & 2,3 & 2,1 & 1 \\
\hline $\begin{array}{l}\text { Instruments } \\
\text { de précision }\end{array}$ & 21,1 & 2,2 & 21,4 & 1 \\
\hline $\begin{array}{l}\text { Composants } \\
\text { électroniques }\end{array}$ & 17,1 & 1,8 & 25,6 & 1 \\
\hline
\end{tabular}

Note: ${ }^{a}$ Équivalent prix. Par exemple, un chiffre de 2 signifie que dans le secteur considéré, les exportateurs peuvent avoir des prix plus de deux fois supérieurs à ce qu'ils seraient si leur qualité était identique à la moyenne des pays de l'OCDE.

Sources: Calculs des auteurs, cf. Bas, Martin et Mayer (2014) op. cit. et OMC.

Les trois secteurs français les plus compétitifs sur la dimension hors prix sont l'aéronautique, la maroquinerie et le vin. En Allemagne, ce sont les pièces détachées automobile, les métaux non ferreux et les produits plastiques. Ces trois premiers secteurs représentent $15 \%$ des exportations allemandes, contre seulement $7 \%$ des exportations françaises. Par ailleurs, l'Allemagne est $n^{\circ} 1$ de l'OCDE sur ces trois secteurs, tandis que la France est $n^{\circ} 2$ sur la maroquinerie et $\mathrm{n}^{\circ} 3$ sur le vin. Lorsqu'on descend dans la liste, l'Allemagne reste $n^{\circ} 1$ pour ses dix premiers secteurs, tandis que la France descend au $8^{\mathrm{e}}$ rang de l'OCDE pour son dixième secteur (produits plastiques). L'Allemagne se situe donc clairement devant la France en termes de compétitivité hors prix. Ceci est d'autant plus pénalisant pour les exportations françaises que parmi les dix secteurs les plus compétitifs sur le critère hors prix, quatre sont communs aux deux pays : l'Allemagne est notre plus proche concurrent qui nous dépasse en termes de compétitivité hors prix.

On étudie maintenant, pour chaque secteur, les dix meilleurs pays en termes de compétitivité hors prix. En 2013, la France 
place 55 secteurs (sur 102 analysés) dans le «top 10 » de l'OCDE (graphique 3), ce qui la situe au $7^{\mathrm{e}}$ rang de l'OCDE, une position relativement bonne (et stable) ${ }^{18}$. Le graphique montre que c'est l'Allemagne qui se détache, avec 85 secteurs dans le « top 10 ». L'Italie, la Suisse, les Pays-Bas, le Royaume-Uni et le Japon devancent également la France mais dans une moindre mesure (entre 57 et 65 produits). Les États-Unis font légèrement moins bien que la France ( 51 produits).

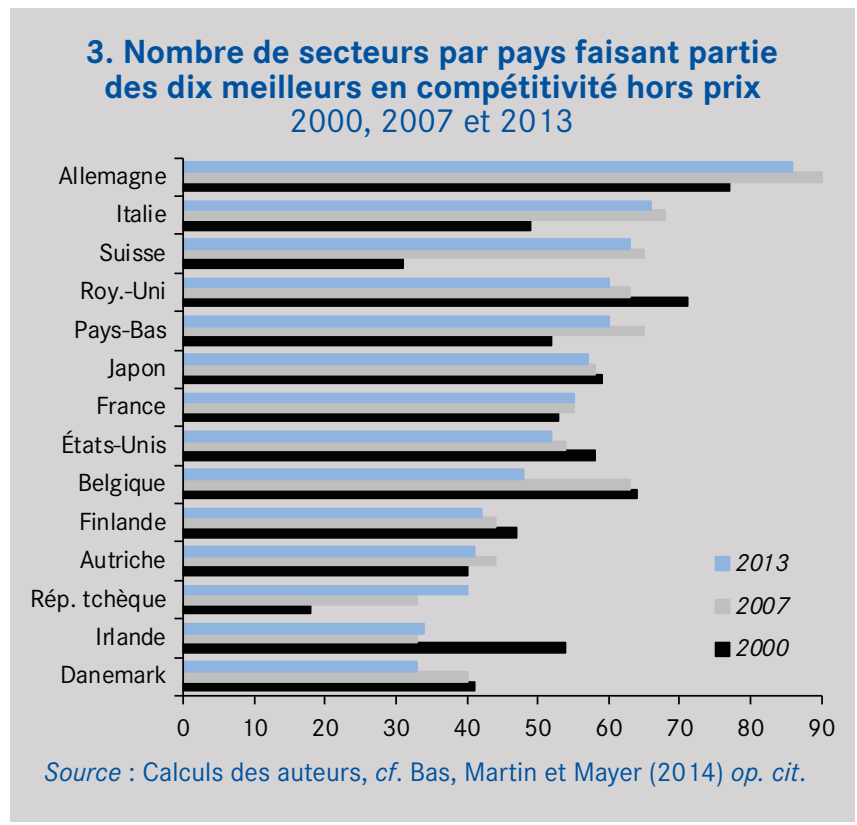

Le graphique 4 a décompose les variations de parts de marché de quelques pays de l'OCDE en variations dues à la compétitivité prix et hors prix, sur la période 2000-2013. On examine ici les parts de marché relatives à la moyenne de I'OCDE, donc entre pays comparables. Ce graphique oppose, d'un côté, le Japon, le Royaume-Uni et les États-Unis, qui ont perdu davantage de parts de marché que la moyenne de l'OCDE, et, de l'autre, l'Allemagne et surtout la Pologne, qui ont perdu moins de parts que la moyenne, voire en ont gagné (cas de la Pologne et plus généralement des pays d’Europe centrale et orientale, du Mexique et de la Turquie, non représentés sur le graphique). Dans les deux cas, les évolutions de parts de marché semblent s'expliquer essentiellement par la compétitivité hors prix, qui régresse dans le premier groupe et augmente dans le second. La France se situe dans une position intermédiaire, qui cache néanmoins des évolutions contrastées entre secteurs ${ }^{19}$ et au cours du temps.

\section{... mais décroche dans plusieurs secteurs depuis 2008}

De 2000 à 2007, les parts de marché françaises baissent un peu moins rapidement que la moyenne de l'OCDE

\section{Variations annuelles des parts de marché relatives et composante compétitivité prix et hors prix, en \%}

\section{a. $2000-2013$}

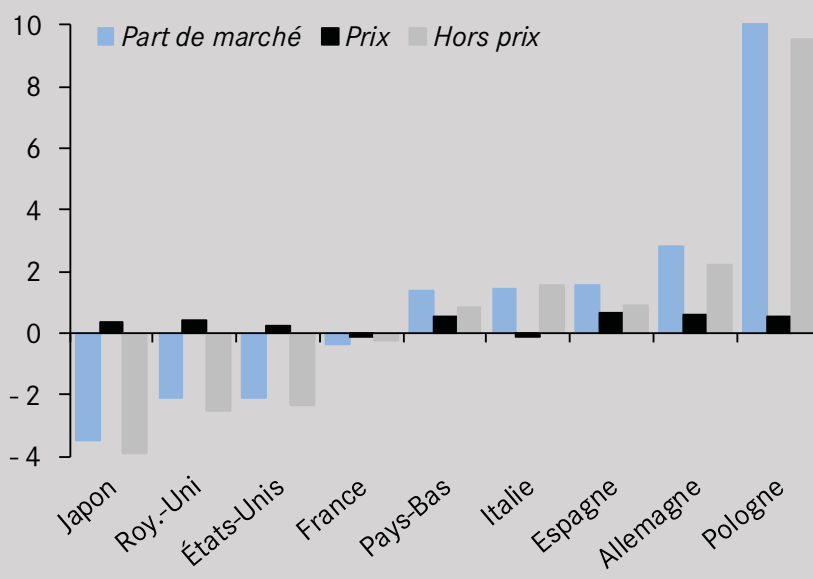

b. $2000-2007$

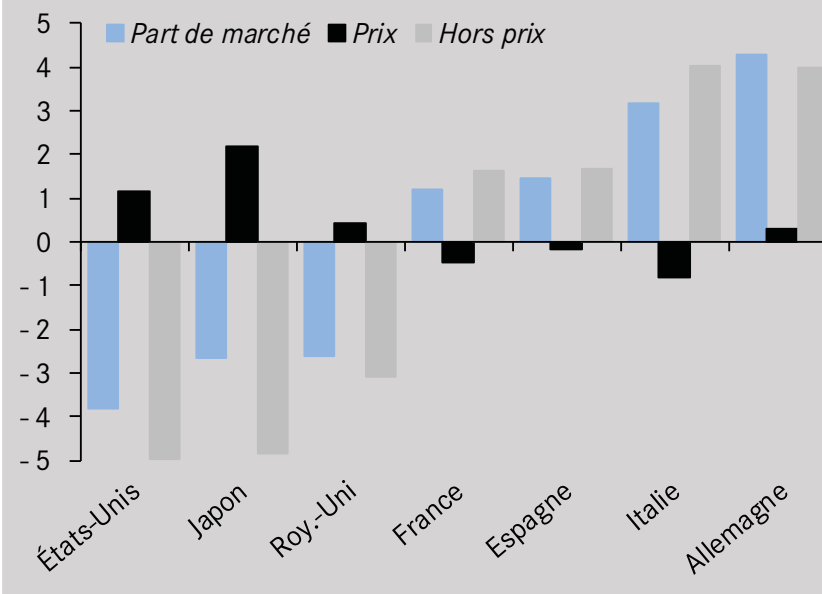

c. $2008-2013$

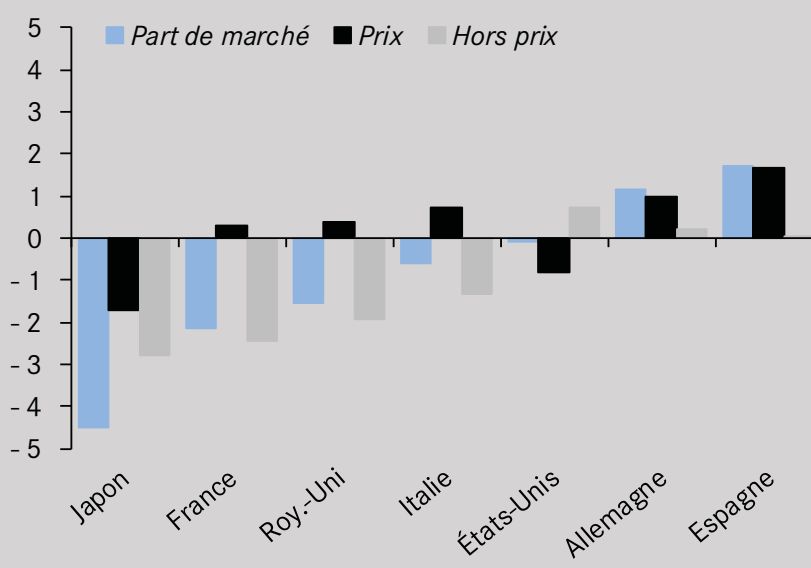

Lecture : Évolution relative par rapport à la moyenne de l'OCDE. Source: Calculs des auteurs, cf. Bas, Martin et Mayer (2014) op. cit.

${ }^{18}$ Le constat de valeurs unitaires d'exportation relativement élevées dans le cas français est confirmé par Fontagné L., G. Gaulier et S. Zignago (2008) : « Specialization Across Varieties and North-South Competition ॥, Economic Policy, vol. 23, n 53, pp.51-91. Les auteurs s'intéressent au commerce extra-européen.

${ }^{19}$ Les deux meilleurs secteurs français en termes de compétitivité hors prix - l'aéronautique et la maroquinerie - ont enregistré de fortes augmentations de cette compétitivité depuis 2000. Voir l'évolution de la compétitivité hors prix pour plusieurs secteurs français dans le focus sectoriel en ligne, www.cae-eco.fr 
(graphique 4b). Durant cette période, la compétitivité prix française se dégrade (en partie du fait de l'appréciation de l'euro), mais ceci et compensé par une nette augmentation de la compétitivité hors prix. Durant cette période, l'Allemagne améliore sa compétitivité prix grâce à la modération salariale et à la délocalisation des segments les moins compétitifs de sa chaîne de production. Mais l'augmentation très marquée de sa compétitivité hors prix montre que l'Allemagne a utilisé cette période pour augmenter la qualité de ses produits. Ce constat relativise l'idée selon laquelle l'Allemagne a mené une stratégie fondée uniquement sur la baisse des coûts relatifs durant cette période.

Sur la période 2008-2013, la France a une performance bien inférieure à la moyenne OCDE, alors même que sa compétitivité prix s'améliore un peu (graphique 4c). La compétitivité hors prix se dégrade fortement. II est possible que l'amélioration de la compétitivité hors prix avant 2008 ait résulté essentiellement d'un effet de sélection : compte tenu de la dégradation de la compétitivité prix au cours de cette période, seuls les exportateurs les plus productifs et ceux avec une compétitivité hors prix élevée ont pu survivre, ce qui est cohérent avec la baisse du nombre d'exportateurs enregistrée jusqu'en 2009 (graphique 2) et la baisse des taux de marge dans l'industrie ${ }^{20}$. À partir de 2008, alors que la compétitivité coût de la France se redresse légèrement, le manque d'innovation, d'investissement et de montée en gamme (difficiles dans une situation de faibles marges) semble amener à un décrochage de la compétitivité hors prix. Tout en restant dans le "top 10 », plusieurs secteurs sont en recul pour la France : appareils de distribution électrique, vin, pièces détachées automobile, ameublement.

Constat 4. Parmi les pays de l'OCDE, la France jouit d'une compétitivité hors prix relativement bonne ( $7^{\mathrm{e}}$ rang de l'OCDE) mais cette compétitivité a régressé depuis 2008. L'Allemagne a une position extrême parmi les pays OCDE.

\section{Comment redresser les parts de marché ?}

Le débat sur la compétitivité a tendance à se focaliser sur des explications secondaires à la contre-performance française à l'exportation (mauvaise spécialisation géographique ou sectorielle, contraintes de crédit ou insuffisance des aides aux exportateurs) et à accorder une place insuffisante au facteur le plus important quantitativement : le rapport qualité-prix des produits.

\section{La compétitivité prix}

La compétitivité prix s'appréhende de manière différente selon qu'on s'intéresse aux marchés de la zone euro ou hors zone euro, même s'il faut relativiser cette distinction dans la mesure où la France est en concurrence avec des pays de la zone euro sur des marchés hors zone euro.

\section{Le taux de change}

La compétitivité sur les marchés hors zone euro est fortement dépendante du taux de change de l'euro. Les estimations sur données d'entreprises françaises suggèrent que, toutes choses égales par ailleurs, une dépréciation de $10 \%$ de l'euro par rapport à un pays partenaire hors zone euro relève la valeur des ventes d'un exportateur moyen vers ce pays d'environ 5-6\%. Cette augmentation - qui a lieu pour l'essentiel l'année même de la dépréciation - vient principalement d'une hausse des volumes exportés (4-5\%), le reste (0,5-1\%) provenant d'une revalorisation des marges sur chaque unité vendue (via une légère hausse de prix en euro) ${ }^{21}$. Au niveau agrégé, l'impact d'une dépréciation de $10 \%$ de l'euro sur la valeur des exportations est plus important - de l'ordre de 7-8 \% - car la dépréciation non seulement améliore la situation des exportateurs déjà en place, mais elle fait aussi entrer de nouvelles entreprises sur les marchés export. De mars 2014 à mars 2015, le taux de change effectif réel de la France s'est déprécié d'environ $6 \%$. Une simple règle de trois suggère que nos exportations devraient en être augmentées d'environ 4-5\%.

\section{Travail et consommations intermédiaires}

Quels sont alors les éléments de coût sur lesquels la politique économique française peut faire la différence par rapport aux concurrents de la zone euro ? Le débat a tendance à se focaliser sur les coûts directs du travail dans les secteurs exportateurs, alors qu'une décomposition de la valeur ajoutée des exportations montre que seulement $23 \%$ de la valeur des exportations sont imputables au coût du travail du secteur exportateur directement concerné. Le coût du travail des autres branches utilisées en consommations intermédiaires domestiques représente $21 \%$ de la valeur des exportations. Les $56 \%$ restants proviennent des consommations intermédiaires (hors le coût du travail des consommations intermédiaires domestiques), parmi lesquels les produits importés ( $25 \%$ de la valeur des exportations). Ces chiffres sont une invite à s'intéresser aux importations comme moyen d'optimiser les chaînes de valeur, à augmenter sans à-coups le prix de l'électricité qui pèse lourdement sur certaines exportations ${ }^{22}$ et, enfin, à maîtriser les prix des services qui ont fortement augmenté depuis dix ans par rapport

\footnotetext{
${ }^{20}$ Voir Martin, J. et I. Méjean (2014) : "Low-Wage Countries' Competition, Reallocation Across Firms and the Quality Content of Exports ॥, Journal of International Economics, vol. 93, n 1, pp. 140-152. Aghion, Ph., Cette, G. et E. Cohen (2014), Changer de Modèle, Odile Jacob.

${ }^{21}$ Voir Bénassy-Quéré, A., Gourinchas, P.O., Martin, Ph. et G. Plantin (2014) : « L'euro dans la guerre des monnaies », Note du Conseil d’analyse économique, $\mathrm{n}^{\circ} 11$, janvier.

${ }^{22}$ Voir Bureau D., L. Fontagné et P. Martin (2013) : «Énergie et compétitivité », Note du Conseil d'analyse économique, $n^{\circ}$ 6, mai.
} 
à l'Allemagne du fait du coût du travail, mais aussi du niveau encore élevé des obstacles à la concurrence dans les secteurs de services ${ }^{23}$.

Plus généralement, il faut souligner que l'ensemble de l'économie française participe à la construction de la compétitivité prix. Améliorer le fonctionnement du marché du logement, par exemple, contribue à la compétitivité dans la mesure où la hausse des loyers et de l'immobilier pèse sur le budget des ménages et conduit à terme à des hausses de salaires. On peut étendre le raisonnement à tous les services du secteur abrité, y compris les administrations publiques : toute l'économie française est concernée par la question de la compétitivité prix, pas seulement les entreprises exportatrices qui sont en première ligne.

\section{Le ciblage du CICE}

Le Crédit d'impôt compétitivité emploi (CICE) dont la mise en place a débuté en 2013, à la suite de la remise du rapport Gallois sur la compétitivité de l'industrie française ${ }^{24}$, a pour objectif explicite de stimuler l'emploi et d'améliorer la compétitivité des entreprises. Nous nous concentrons ici sur ce second objectif.

Les 20 milliards d'euros annuels du CICE représentent en principe un allègement global du coût du travail de $3 \%$ pour les entreprises concernées. Les entreprises exportatrices ont alors le choix entre : abaisser leurs prix à l'exportation et donc augmenter leur compétitivité prix et leurs parts de marché, relever leurs marges et augmenter les salaires. On manque encore de recul pour savoir comment les entreprises françaises ont réagi, même si des résultats d'enquêtes apportent un premier éclairage ${ }^{25}$. Mais on sait que le CICE couvre des salaires allant jusqu'à 2,5 fois le SMIC, seuil choisi de manière à toucher les entreprises de l'industrie qui réalisent l'essentiel des exportations et où les salaires sont en moyenne plus élevés que dans les services. À ces niveaux de rémunération, les salariés (qualifiés) font face à un risque de chômage relativement faible: les actifs avec un niveau d'études au-dessus du Bac font face à un taux de chômage voisin de $5 \%$. Dans ces conditions, une exonération réduisant a priori le coût du travail de $1 \%$ pourrait se traduire par un accroissement des salaires de $0,9 \%{ }^{26}$.
A posteriori, la baisse du coût du travail ne serait donc que de $0,1 \%$ pour les salariés subissant un taux de chômage faible. Le gain de compétitivité prix ou l'augmentation des marges seraient alors limités. Ces résultats suggèrent d'évaluer l'impact du CICE sur la dynamique des salaires et, au cas où cette analyse montrerait une plus forte croissance des salaires au-dessus de 1,5 SMIC après la mise en place du CICE, de recentrer le bénéfice du CICE et du pacte de responsabilité sur les salaires inférieurs à 1,5 SMIC.

Le contre-argument généralement avancé est que les allègements de charges sur les bas salaires bénéficient principalement à des secteurs abrités de la concurrence internationale - commerce de détail, services à la personne, par exemple. Les entreprises exportatrices, généralement dans l'industrie, emploient davantage de travailleurs à relativement haut salaire. En ne touchant pas les hauts salaires, le CICE raterait son objectif de compétitivité. Cependant, comme on l'a vu, une proportion importante de la valeur des exportations est constituée de services incorporés, dont une part de services peu qualifiés (nettoyage, gardiennage, restauration, transport). La baisse du coût du travail peu qualifié est donc une composante importante de la compétitivité prix.

\section{Recommandation 1. Évaluer l'impact du CICE sur la dynamique des salaires. Si une plus forte croissance des salaires relativement élevés est constatée après la mise en place du CICE, concentrer les allègements de charges sociales sur les bas salaires.}

\section{La compétitivité hors prix}

Les politiques de compétitivité hors prix se confondent en grande partie avec les politiques de productivité qui passent elles aussi par une stimulation de l'innovation et de montée en gamme. C'est pourquoi on peut reprendre ici les préconisations du CAE relatives à la formation et à la recherche ${ }^{27}$. C'est en particulier le cas de la formation professionnelle qui doit aussi être considérée comme un investissement pour augmenter la compétitivité et la montée en gamme.

\footnotetext{
${ }^{23}$ Voir Conseil d'analyse économique (2014) : «Quelles réformes pour la France ? Les préconisations du CAE », Note du Conseil d’analyse économique, $n^{\circ} 15$, juillet.

${ }^{24}$ Gallois L. (2012) : Pacte pour la compétitivité de l'industrie française, Rapport pour le Premier ministre, La Documentation française.

${ }^{25}$ Cf. Enquête de conjoncture de l'INSEE sur l'utilisation du CICE (2014). En général, les entreprises annoncent qu'elles utiliseront le CICE pour augmenter leur résultat d'exploitation ; pour $58 \%$ des entreprises de l'industrie et $52 \%$ des entreprises des services, ce surcroît de ressources sera destiné majoritairement à l'investissement.

${ }^{26}$ Cahuc P. et S. Carcillo (2014) : Alléger le coût du travail pour augmenter l'emploi : les clés de la réussite, Institut Montaigne. Voir aussi Plane M. (2012) : "Évaluation de l'impact économique du CICE », Revue de l'OFCE, n 126, ou Bock S., P. Lissot et S. Ozil (2015) : « Matis : une maquette d'évaluation des effets sur l'emploi de variations du coût du travail ॥, Document de Travail de la DG Trésor, $\mathrm{n}^{\circ}$ 2015/02, mars, qui retiennent un profil décroissant de l'élasticité de l'emploi à son coût suivant le niveau de salaire.

${ }^{27}$ Artus P., C. García-Peñalosa et P. Mohnen (2014) : «Redresser la croissance potentielle de la France », Note du Conseil d’analyse économique, n 16 septembre ; Fontagné L., P. Mohnen et G. Wolff (2014) : «Pas d’industrie, pas d'avenir ? », Note du Conseil d’analyse économique, n 13 , juin.
} 
Cependant, comme le montre l'exemple du luxe, les marques jouent un rôle spécifique à l'exportation en compensant un désavantage de coût et en effaçant le handicap de la distance géographique. Les questions de propriété intellectuelle doivent être prioritaires dans les négociations commerciales internationales dans lesquelles l'Europe est engagée, et cela doit être affirmé clairement par les autorités françaises. Le rejet de I'ACTA (Anti-Counterfeiting Trade Agreement) par le Parlement européen en juillet 2012 est de ce point de vue particulièrement préoccupant, contrastant avec sa signature dès 2011 par le Canada, les États-Unis, le Japon, la Corée, Singapour, la Nouvelle-Zélande et le Maroc.

Recommandation 2. Faire de la défense de la propriété intellectuelle une priorité dans les négociations internationales.

Cependant, la productivité n'est pas seulement affaire de design et d'innovation. Des travaux récents suggèrent qu'une part importante (entre un quart et un tiers) des différences de productivité entre les entreprises et les pays est simplement due à la qualité de la gestion des entreprises ${ }^{28}$. La France se situerait au $7^{\mathrm{e}}$ rang des pays de l'OCDE en termes de qualité moyenne de la gestion de ses entreprises. Par rapport à des pays comme l'Allemagne ou les États-Unis, elle est cependant caractérisée par une plus forte proportion de petites entreprises avec une qualité de gestion plus faible en moyenne et donc aussi une productivité plus faible.
Enfin, la croissance de la productivité, et donc de la compétitivité d'un pays, provient pour une grande partie (pour moitié selon des études récentes) de la réallocation des facteurs de production des entreprises peu productives vers les entreprises plus productives. Les rigidités sur les marchés du travail et des biens freinent cette réallocation ${ }^{29}$. Mesuré au niveau des entreprises, au sein de chaque secteur de l'industrie française, l'écart moyen entre salaire et productivité a augmenté des près de 15 $\%$ en termes réels sur la période $2000-2007^{30}$. Le phénomène d'entreprises ne fonctionnant pas à la taille optimale est plus marqué au voisinage de 50 salariés, reflétant un effet de seuil. Mais la dégradation observée n'est pas plus marquée au voisinage de ce seuil. D'autres sujets doivent donc être considérés comme les coûts de licenciement, le droit des faillites, mais aussi les réglementaions des marchés de biens et services ${ }^{31}$.

\section{Recommandation 3. Dans les arbitrages sur les réformes dites structurelles, prendre explicitement en considération l'impact de ces réformes sur la réallocation des facteurs de production (travail et capital) vers les entreprises les plus productives.}

On voit finalement que la performance à l'export est en grande partie le simple reflet de l'efficacité du système productif national, de sorte que les politiques de compétitivité se confondent avec les politiques destinées à améliorer la productivité.

\footnotetext{
${ }^{28}$ Voir Bloom N., R. Renata Lemos, D. Sadun et J. Van Reenen (2014) : « The New Empirical Economics of Management », CEP Occasional Paper, ${ }^{\circ} 41$.

${ }^{29}$ Aghion et al. (2008) mettent en évidence un lien entre rigidités sur les marchés du travail et des biens et productivité. Dostie et al. (2009) suggèrent que les phénomènes de réallocation des facteurs de production peuvent expliquer entre 50 et $70 \%$ de la croissance de la productivité globale des facteurs. Voir Aghion P., P. Askenazy, R. Bourlès, G. Cette et N. Dromel (2008) : "Distance à la frontière technologique, rigidités de marché, éducation et croissance ", Économie et Statistique, vol. 419, $\mathrm{n}^{\circ}$ 1, pp. 11-30. Bérubé C., B. Dostie et L. Vihuber (2013) : Estimation de la contribution de la réallocation de la main d'œuvre à la croissance de la productivité au Canada, Centre sur la productivité et la prospérité, HEC Montréal, septembre.

${ }^{30}$ Fontagné L. et G. Santoni (2015) : Firm Level Allocative Inefficiency: Evidence from France, Mimeo.

${ }^{31}$ Une étude récente du FMI montre que les réglementations qui réduisent la concurrence sur le marché des biens et services ont un impact négatif sur la croissance de la productivité. Voir Fonds Monétaire International (2015) : World Economic Ooutlook, pp. 104-107.
}

\section{conseil d'analyse économique}

Présidente déléguée Agnès Bénassy-Quéré

Secrétaire générale Hélène Paris

Conseillers scientifiques

Jean Beuve, Clément Carbonnier,

Manon Domingues Dos Santos

Aurélien Eyquem

Assistante de recherche

Alice Keogh

Le Conseil d'analyse économique, créé auprès du Premier ministre, a pour mission d'éclairer, par la confrontation des points de vue et des analyses de ses membres, les choix du Gouvernement en matière économique.

Membres Agnès Bénassy-Quéré, Antoine Bozio, Pierre Cahuc, Brigitte Dormont, Lionel Fontagné, Cecilia García-Peñalosa, Augustin Landier, Philippe Martin, Pierre Mohnen, Xavier Ragot, Jean Tirole, Alain Trannoy, Étienne Wasmer, Guntram Wolff

Correspondants

Anne Perrot, Christian Thimann
Les Notes du Conseil d'analyse économique ISSN 2273-8525

Directrice de la publication Agnès Bénassy-Quéré Rédactrice en chef Hélène Paris

Réalisation Sophie de Salée

Contact Presse Christine Carl

christine.carl@cae-eco.fr Tél. : 0142757747 